Annales Geophysicae (2003) 21: 881-891 (C) European Geosciences Union 2003

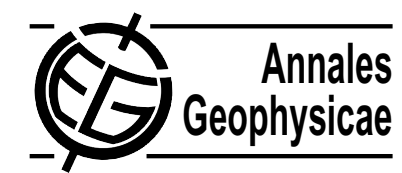

\title{
Generation of Bernstein waves by ion shell distributions in the auroral region
}

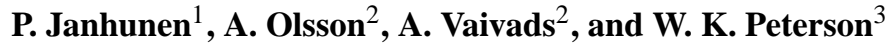 \\ ${ }^{1}$ Finnish Meteorological Institute, Geophysical Research, Helsinki, Finland \\ ${ }^{2}$ Swedish Institute of Space Physics, Uppsala Division, Uppsala, Sweden \\ ${ }^{3}$ LASP, University of Colorado, Boulder, Colorado, USA
}

Received: 14 March 2002 - Revised: 12 September 2002 - Accepted: 15 October 2002

\begin{abstract}
Hot ion shell distributions could possibly contain enough free energy for waves that could power electron energisation above auroral inverted-V regions. Using both linear theory (WHAMP) and two-dimensional electrostatic simulations, we show that ion shell distributions can cause unstable ion Bernstein mode emissions with high temporal growth rates, as well as perpendicular and parallel e-folding distances, that are in accordance with the tranverse dimensions of auroral arcs and the parallel size of the energisation region, respectively. The phase velocities of the waves are in the proper range to give parallel energisation to electrons with a Landau resonance. The simulation shows that about $90 \%$ of the energy goes into electrons and $10 \%$ goes into cold ion perpendicular heating. An electron heating rate of $\sim 80$ $\mathrm{eV} / \mathrm{s}$ is obtained.
\end{abstract}

Key words. Ionosphere (auroral phenomena) - Space plasma physics (numerical simulation studies; wave-particle interactions)

\section{Introduction}

The most widely accepted model for the acceleration of electrons to form the inverted-V spectra above stable auroral arcs is the potential drop acceleration model (Carlqvist and Boström, 1970; Evans, 1974). However, it has also been suggested that instead of a static potential drop, plasma waves could be responsible for electron energisation. Lower-hybrid waves (Bingham et al., 1988; Bryant et al., 1991; Bryant and Perry, 1995; Bryant, 1999), as well as Alfvén waves (Goertz and Boswell, 1979; Kletzing, 1994; Genot et al., 2000; Stasiewicz et al., 2000), have been suggested for this purpose. It has been pointed out by Louarn et al. (1994); Volwerk et al. (1996); Wygant et al. (2000) that Alfvén waves contain enough downward Poynting flux to power the aurora in at least some events during disturbed conditions. It was later suggested (Janhunen and Olsson, 2000) that both wave-

Correspondence to: A. Olsson (ao@irfu.se) particle interactions and a potential structure can accelerate electrons in cooperation. In this model the waves need to energise electrons in the parallel direction only to some hundreds of eV energy, since the potential structure takes care of producing a several $\mathrm{keV}$ inverted- $\mathrm{V}$ spectrum at low altitude.

In this paper we suggest that ion Bernstein waves could be responsible for such wave-induced electron energisation. We approach the question by first identifying an energy source that contains enough free energy (the ion shell distribution) and then consider what wave modes the energy source can make unstable by applying linear analysis and particle simulation. Particle and wave observations from Polar are used to constrain the model.

\section{Shell distributions}

Ionic free energy sources, such as temperature anisotropies $\left(T_{\perp}>T_{\|}\right)$and drifts and loss cones, have previously been considered to excite ion waves near the ion cyclotron frequency (André, 1986; André et al., 1986). However, considering the energy needed to power the inverted- $\mathrm{V}$ electron acceleration in an auroral arc (typically a few tens of milliwatts per square meter when projected to ionospheric altitude, or clearly more than that in bright auroras), these free energy sources do not appear to be sufficient. As a possible remedy to this problem we consider a shell distribution, i.e. an isotropic (quasi-Maxwellian) hot ion distribution from which the low-energy core has been partly removed. Theoretical treatment of shell distribution has been given by Sentman et al. (1986) and Freund and Wu (1988). A shell distribution could be generated, for example, by some process near the equatorial plane that energises hot ions in the parallel direction so that a positive slope in the distribution function in the parallel direction is generated (Elphic and Gary, 1990). Due to the conservation of energy and the first adiabatic invariant, such a distribution turns into a shell-like distribution upon reaching lower altitudes. This has been shown for the 


\section{Flattening procedure}

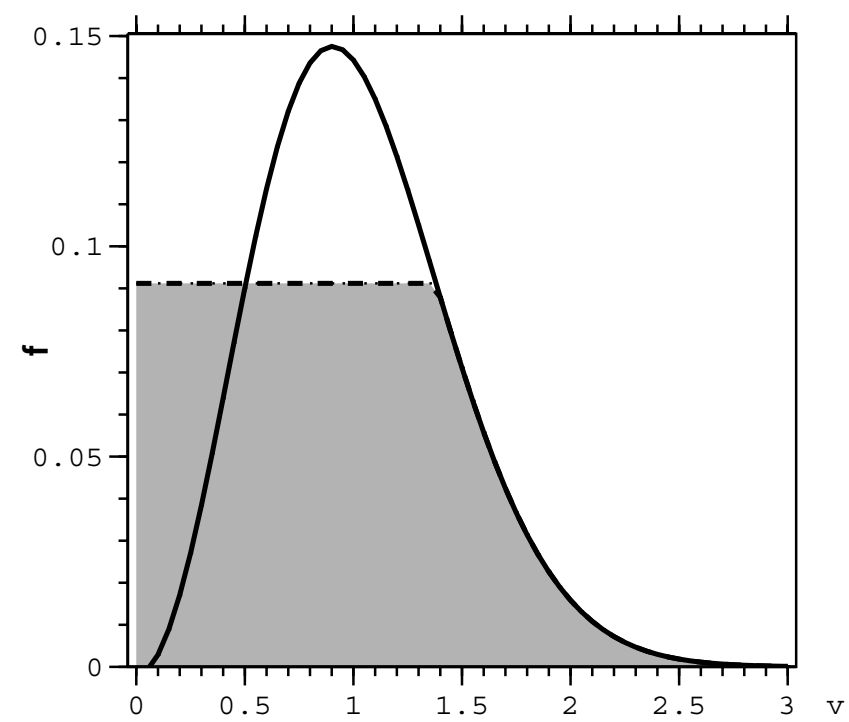

Fig. 1. Example shell distribution generated by the expression $f(v)=\exp \left(-v^{2}\right)-1.00206 \exp \left(-1.5 v^{2}\right)$. The velocity and the distribution function are in arbitrary units. The shaded region delimited by dotted line is the closest equilibrium distribution defined in the text. The free energy is $6.9 \%$ of the total kinetic energy in this case.

electrons by Barbosa (1977), but the same argument holds for ions as well.

We define the free energy of a given distribution function to be the kinetic energy of the original distribution minus the kinetic energy of the "closest" equilibrium distribution (the distribution which would be produced from the original distribution by wave-particle interactions). Since positive slopes in velocity space are sources of plasma instabilities, we assume here that the wave-particle interactions locally "level out" the distribution in places where it has a positive slope, repeat the flattening procedure until no positive slopes are found, and assume that the resulting distribution corresponds to the closest equilibrium distribution.

Figure 1 shows an example of a shell distribution and its closest equilibrium distribution (shaded region delimited by dashed line) found by the flattening procedure. In this case the free energy is $6.9 \%$ of the total kinetic energy. This kind of shell distribution was constructed from a difference of two Maxwellians. If the shell distribution is constructed by other means, for example, by simply cutting away the centre of a Maxwellian, much higher free energies (25\% and more) can be reached.

This definition of the free energy is only an upper limit of the physically available free energy. Our numerical simulations indicate that the way of estimating the free energy as defined above is adequate at least to obtain a starting point for numerical investigations. Thus, a shell distribution may contain much more free energy than, for example, an ion loss cone distribution with a $5^{\circ}$ to $10^{\circ}$ loss cone.

To discuss the powering of auroral arcs we need to estimate the energy flux. Let us suppose that the process of extracting the free energy from the shell distribution takes place at some altitude and that the shell distribution is continuously replenished by downgoing new particles at that altitude. The maximum possible downward energy flux is then $v u$, where $v$ is the velocity of the ions forming the shell and $u$ is the free energy density of the shell distribution. (This maximum energy flux is not reached in the example given below in Fig. 3 because there the upgoing part still has free energy left, but the point here is to make a rough estimation only.) For example, if $u=0.5 \mathrm{keV} \mathrm{cm}^{-3}$ (as it is in the example below, Fig. 2) and $v \sim 1.4 \cdot 10^{6} \mathrm{~m} \mathrm{~s}^{-1}$ (i.e. a shell consisting of 10 $\mathrm{keV}$ protons), we obtain an energy flux of $13 \mathrm{~mW} \mathrm{~m}^{-2}$ when projected down to the ionosphere from $5 R_{E}$ radial distance. Although this is an upper limit of the possible energy flux, it shows that there is potentially enough free energy in the shell distribution to power typical auroral arcs.

In addition to the spherical shell distribution considered in this paper, waves that become unstable by a ring (i.e. toroidal) ion distribution have been considered in the cusp by several authors (e.g. Huddleston et al., 2000; Roth and Hudson, 1985).

A shell distribution with a loss cone has been considered earlier and found to produce unstable lower hybrid waves (Bingham et al., 1999). The shell distribution with a loss cone was called the "horseshoe distribution" by Bingham et al. (1999).

\section{Observations}

In Fig. 2 we show an example of an ion shell distribution event in the auroral region at about $21000 \mathrm{~km}$ altitude using Polar. We use data from the electric field instrument (Harvey et al., 1995), TIMAS ion detector (Shelley et al., 1995) and HYDRA electron instrument (Scudder et al., 1995). The free energy enhancement in the ion distribution at 09:3109:34 UT (panel j) occurs close to electrostatic waves (panels b-d), simultaneously with an upward ion conic (panel g) and close to electron middle-energy $T_{\|} \gg T_{\perp}$ type anisotropy (panels $h$ and i). Panel (h) is the ratio of the parallel versus perpendicular electron distribution function (i.e. anisotropy) colour-coded so that red means that there are more parallel than perpendicular electrons in the given energy, whereas panel (i) quantifies the electron middle-energy anisotropy in density units by showing the parallel minus perpendicular distribution function integrated in the region of velocity space corresponding to energies between $100 \mathrm{eV}$ and $1 \mathrm{keV}$ by a red line and similarly the up minus the down difference by a green line. Figure 3 shows the detailed ion distribution function at 09:32 UT, clearly showing a shell distribution. In this example the positive slope of the shell distribution is at $\sim 6 \mathrm{keV}$. Simultaneously with the high energy shell, some low energy $(<1 \mathrm{keV})$ ions also occur. A decomposition into $\mathrm{H}+$ and $\mathrm{O}+$ reveals that the shell is purely 


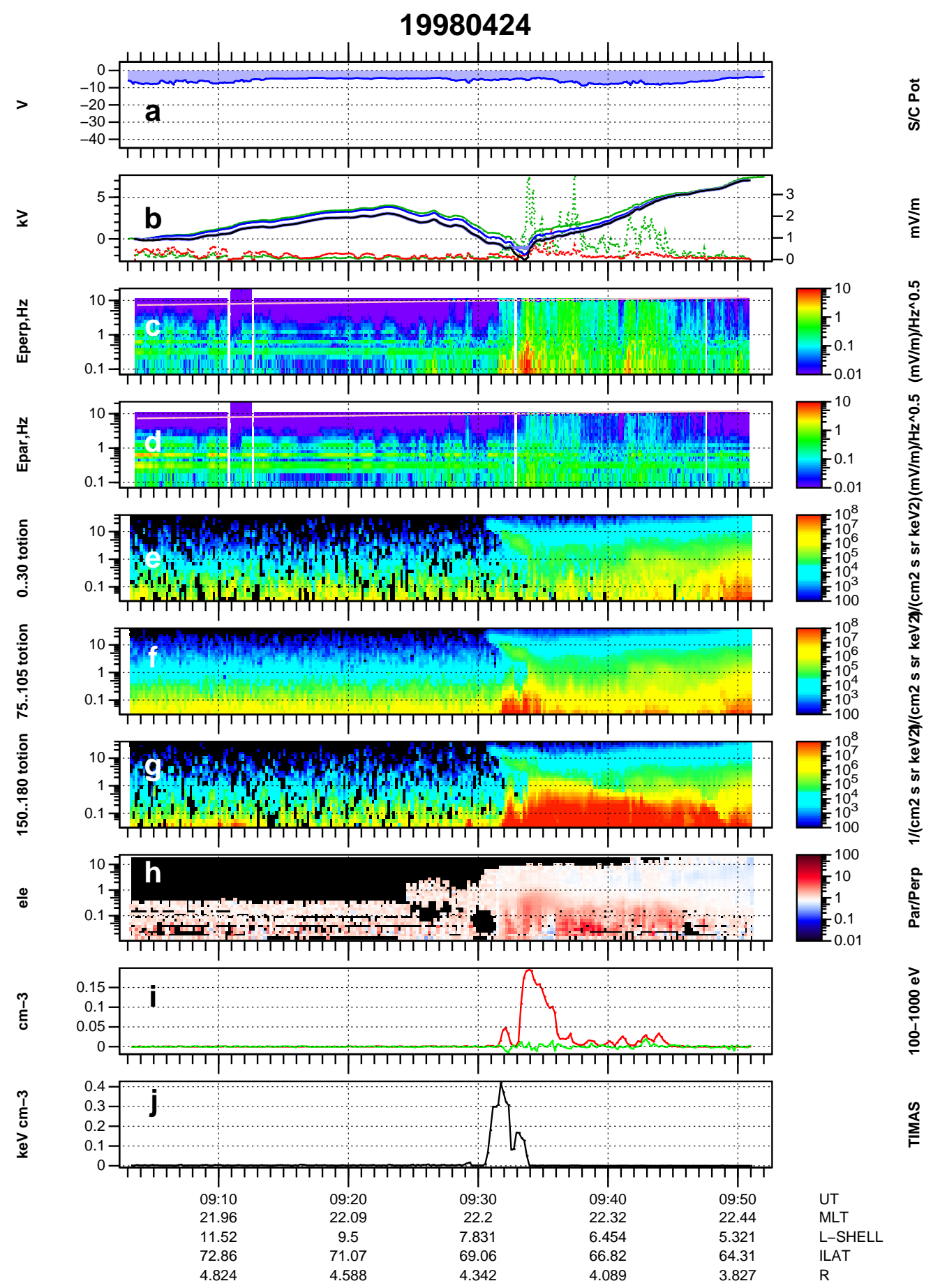

Fig. 2. Polar data for 24 April 1998, 09:05-09:55 UT. Panels are named (a)-(j) from top to bottom. (a) Spacecraft potential from the electric field instrument (EFI), (b) EFI Perpendicular electric wave amplitude in frequency range 1-10 Hz as 12-s average (green), parallel wave amplitude (red), plasma potential integrated along satellite trajectory (black), (c) perpendicular wave electric field spectrogram, (d) parallel wave electric field spectrogram, (e) total ion distribution function from TIMAS for 0-30 ${ }^{\circ}$ pitch angle electrons (downgoing), (f) same for perpendicular ions $\left(75-105^{\circ}\right)$, (g) same for antiparallel (upgoing) ions, $150-180^{\circ}$, (h) ratio of parallel to perpendicular electron distribution function from HYDRA, (i) red line: parallel minus perpendicular electron distribution function integrated between 0.1 and 1 $\mathrm{keV}$ (the anisotropic density), green line: up minus down parallel component, positive means upgoing electrons, (j) free energy density in TIMAS ion distribution function. 


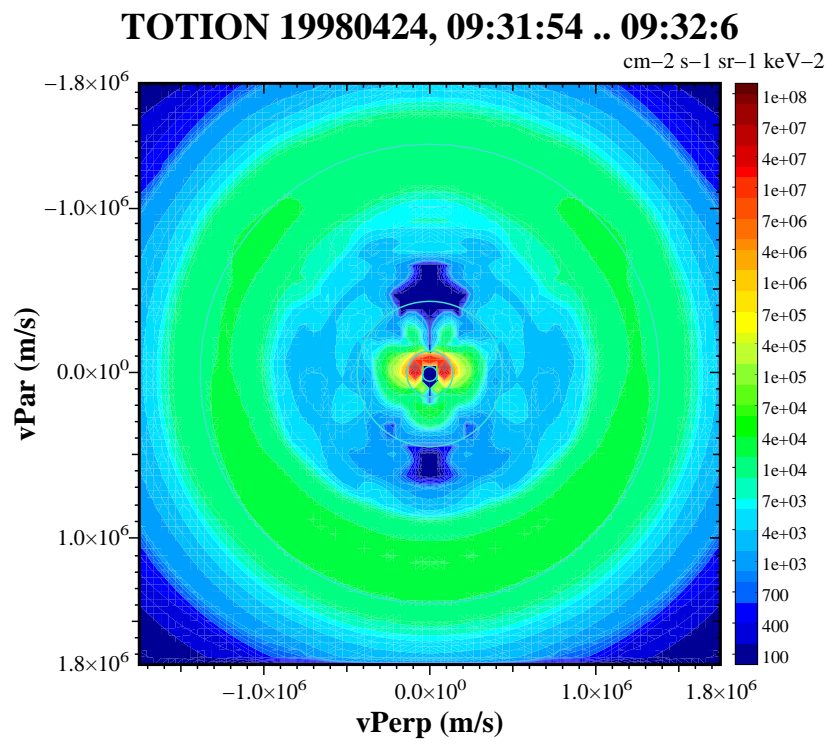

Fig. 3. Total ion distribution function from TIMAS at 09:32 during the event shown in Fig. 2. A clear shell distribution is seen starting from $0.8 \times 10^{6} \mathrm{~m} / \mathrm{s}(3.5 \mathrm{keV})$. The blue circles correspond to energies $10 \mathrm{eV}\left(0.044 \times 10^{6} \mathrm{~m} / \mathrm{s}\right), 100 \mathrm{eV}\left(0.14 \times 10^{6} \mathrm{~m} / \mathrm{s}\right), 1 \mathrm{keV}$ $\left(0.44 \times 10^{6} \mathrm{~m} / \mathrm{s}\right)$ and $10 \mathrm{keV}\left(1.4 \times 10^{6} \mathrm{~m} / \mathrm{s}\right)$.

hydrogen, while the low energy ions are mostly oxygen (plot not shown). Thus, the shell is probably of magnetospheric origin and the low energy ions come from the ionosphere (an upward oxygen conic).

The wave electric fields at around 09:33-09:36 UT are mainly perpendicular, bursty and broad band (Fig. 2, panels $\mathrm{c}-\mathrm{d})$. Such electric fields have been named broad-band electrostatic noise (BEN) and they have been observed on auroral field lines in correlation with the occurrence of inverted-V events (Gurnett and Frank, 1977; Gurnett, 1991). The physical interpretation of BEN is still not certain, but it has been suggested that ion Bernstein modes could be partly involved in the BEN spectrum (Ashour-Abdalla and Thorne, 1978). Afterwards, it has been shown by André (1986), with more extensive WHAMP computations, that ion Bernstein modes can be excited from a hot ion loss cone distribution, even in the presence of some cold plasma.

The electron anisotropies (panels $\mathrm{h}$ and i) are correlated with waves (panels $\mathrm{c}$ and d) but, over a short time at least, almost anticorrelated with the shell distribution free energy (panel j). Inspection of the ion distribution function shows that when the anisotropies appear at 09:34 UT, the H+ distribution function has a plateau at the same energy where a shell distribution existed one minute earlier in the data (Fig. 4). Thus, it is possible that the middle-energy electron anisotropies are due to a wave-particle interaction that takes the free energy from the shell distribution, thus leaving an ion plateau and an electron distribution with enhanced parallel temperature (i.e. the anisotropy).

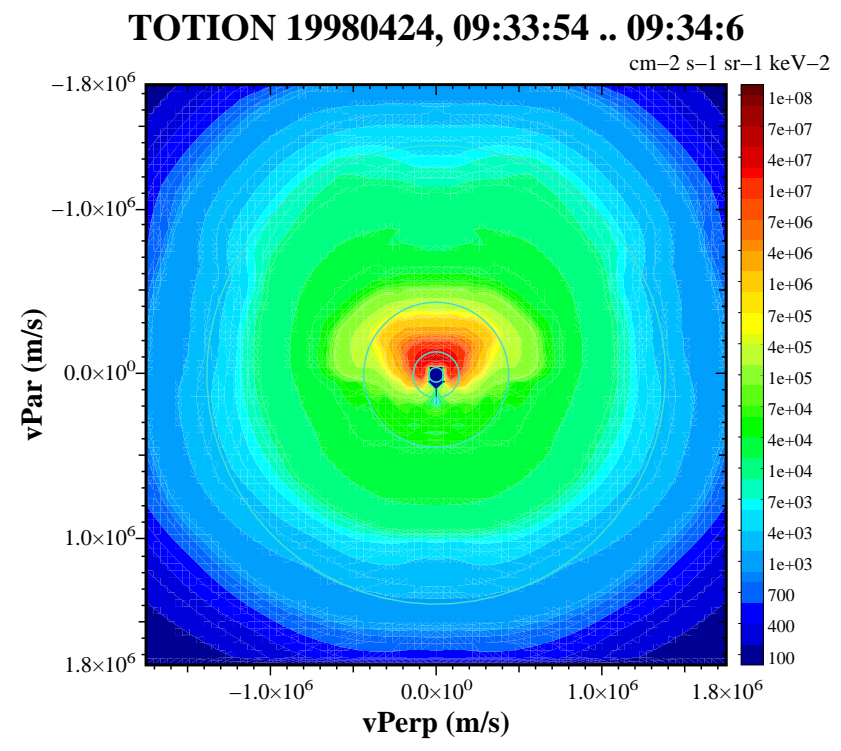

Fig. 4. Total ion distribution function from TIMAS at 09:34 UT during the event shown in Fig. 2. A plateau distribution within 3 $\operatorname{keV}\left(0.75 \times 10^{6} \mathrm{~m} / \mathrm{s}\right)$ with a superposed upward ion conic is seen. The blue circles correspond to energies $10 \mathrm{eV}\left(0.044 \times 10^{6} \mathrm{~m} / \mathrm{s}\right)$, $100 \mathrm{eV}\left(0.14 \times 10^{6} \mathrm{~m} / \mathrm{s}\right), 1 \mathrm{keV}\left(0.44 \times 10^{6} \mathrm{~m} / \mathrm{s}\right)$ and $10 \mathrm{keV}(1.4 \times$ $\left.10^{6} \mathrm{~m} / \mathrm{s}\right)$.

\section{Linear analysis of ion Bernstein modes}

To estimate the linear growth rates of waves we use the computer program WHAMP (Rönnmark, 1982). WHAMP is able to compute the dielectric tensor and solve the linearised dispersion equation for a magnetised plasma whose distribution function can be written as a sum of Maxwellians. We model a shell distribution by a sum of two Maxwellians, one of which has formally a negative density. An example of such a distribution was shown in Fig. 1.

To show that ion Bernstein modes can become unstable by a hot ion shell distribution, we assume that the plasma consists of cold background electrons with density $1 \mathrm{~cm}^{-3}$ and temperature $2 \mathrm{eV}$, cold background protons with density $0.3 \mathrm{~cm}^{-3}$ and temperature $1 \mathrm{eV}$, hot protons with density $0.7(1+c) \mathrm{cm}^{-3}$ and temperature $5 \mathrm{keV}$, and a removed negative proton core with density $-0.7 c \mathrm{~cm}^{-3}$ and temperature $3.33 \mathrm{keV}$. The parameter $c$ controls the strength of the free energy source and is chosen here as $c=1.2$ to give a strong shell distribution. The $5 \mathrm{keV}$ and $3.33 \mathrm{keV}$ populations together model the hot ion shell distribution. Although idealised, this shell distribution is not far from those observed, one example of which was shown in Fig. 3. The magnetic field value of $1000 \mathrm{nT}$ is used, corresponding to $\sim 18000 \mathrm{~km}$ altitude. The electron and ion distribution functions are shown in Fig. 5.

We investigated the seven lowest ion Bernstein modes. The properties of the Bernstein modes at the maximum growth rate found on each surface are summarised in Table 1. The values given in Table 1 are the wave frequency $R_{E} f$, the 
Table 1. Properties of Bernstein waves at region of maximum growth

\begin{tabular}{llllllll}
\hline Parameter & Mode 1 & Mode 2 & Mode 3 & Mode 4 & Mode 5 & Mode 6 & Mode 7 \\
\hline$R_{E} f / \mathrm{Hz}$ & 30 & 45 & 61 & 75 & 92 & 107 & 122 \\
$\lambda_{\perp}(\mathrm{km})$ & 8 & 2.5 & 1.2 & $5.5(7)$ & $4(5.5)$ & $2.5(4.5)$ & $2.6(4)$ \\
$\lambda_{\|}(\mathrm{km})$ & 3000 & 5000 & 5000 & $1000(700)$ & $900(550)$ & $600(450)$ & $300(300)$ \\
$\gamma=2 \pi \operatorname{Im} f(1 / \mathrm{s})$ & 0.16 & 0.03 & 0.017 & $3(1.5)$ & $4.4(2.2)$ & $8(4)$ & $11(5.5)$ \\
Par. e-fold. dist. $(\mathrm{km})$ & 400 & 600 & 600 & $300(400)$ & $300(300)$ & $300(300)$ & $300(600)$ \\
Perp. e-fold. dist. $(\mathrm{km})$ & 8 & 10 & 20 & $3(6)$ & $1(3)$ & $0.6(1)$ & $1(0.3)$ \\
Par. phase vel. $\left(10^{7} \mathrm{~m} / \mathrm{s}\right)$ & 8 & 18 & 30 & $8(5.3)$ & $7(5.2)$ & $6(4.8)$ & $3.9(3.9)$ \\
$E_{\|} / E_{\perp}$ & 0.003 & 0.006 & 0.0003 & $0.004(0.01)$ & $0.006(0.01)$ & $0.005(0.01)$ & $0.009(0.03)$ \\
\hline
\end{tabular}
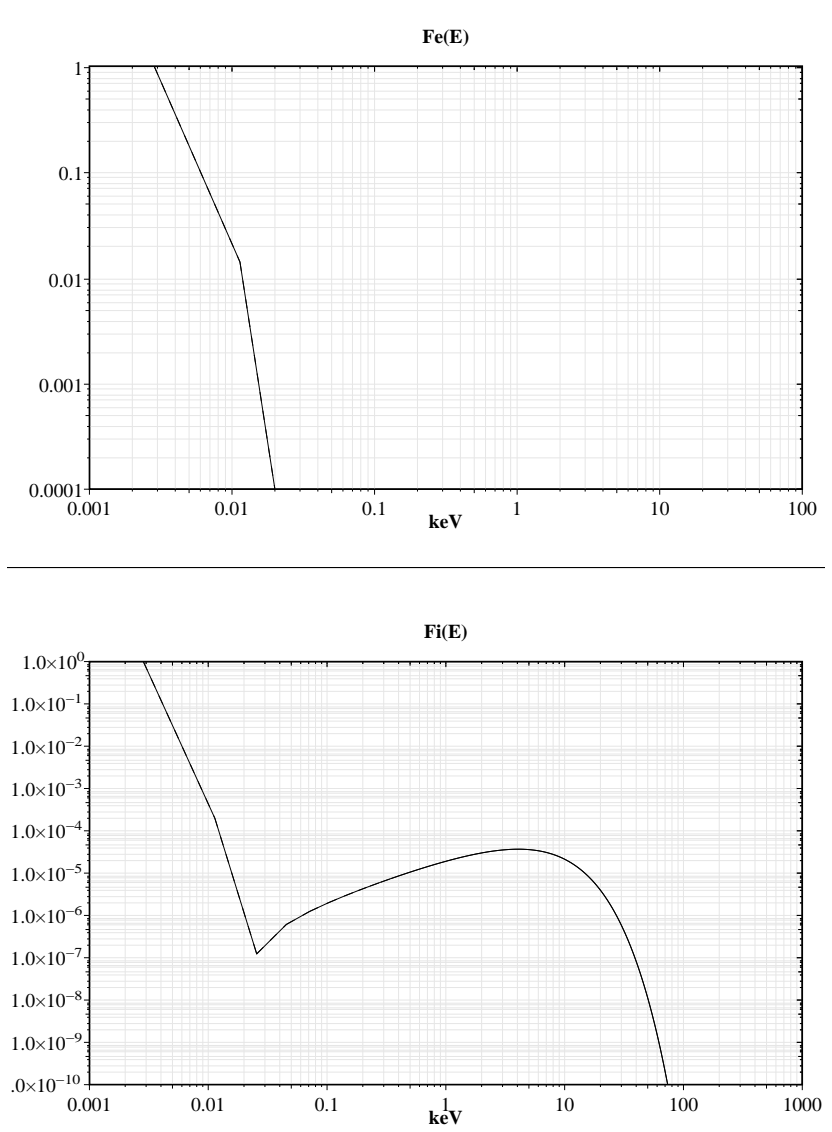

Fig. 5. The electron (upper panel) and ion (lower panel) distribution functions of the plasma model. The vertical axis is in arbitrary units, the maximum being normalised to 1 .

perpendicular wavelength $\lambda_{\perp}$, the parallel wavelength $\lambda_{\|}$, the growth rate $\gamma=2 \pi \operatorname{Im} f$, the parallel $e$-folding distance (the parallel group velocity divided by $\gamma$ ), the perpendicular $e$ folding distance (the perpendicular group velocity divided by $\gamma$ ), the parallel phase velocity, and the parallel versus perpendicular wave electric field. The $E / B$ ratio of the wave fields is not shown but was found to be large, more than $10^{9} \mathrm{~m} / \mathrm{s}$, for all modes at a region of maximum growth, so the waves are nearly electrostatic. For modes $4-7$, the largest $E_{\|} / E_{\perp}$ ratio was searched along the $50 \%$ growth rate contour. The values found in this way appear in parentheses in Table 1. The frequency $f$ is in $\mathrm{Hz}$, thus the $e$-folding time is given by $1 /(2 \pi \operatorname{Im}(f))$.

The first three modes have positive growth rate regions for several thousand kilometres parallel and a few kilometre perpendicular wavelengths. Modes 4-7 have much larger growth rates, appearing at $300-1000 \mathrm{~km}$ parallel and $2.5-5.5$ $\mathrm{km}$ perpendicular wavelengths. Modes above seven seem to be damped for the parameters studied here.

The Bernstein modes 4-7 show some interesting structure. The modes seem coupled due to the presence of hot ions in a somewhat similar manner as found by André (1986), and large growth rates are achieved.

As an example, in Fig. 6, we show the real part of the frequency (black contours) and the temporal growth rate (shading) for the seventh Bernstein mode. Zero, 10, 50, and 90 percent of the maximum growth rate are also displayed (white contours).

As can be seen from Table 1 , the growth rate $\gamma$ is modest for modes 1-3, but large for modes 4-7. The parallel $e$-folding distance is a few hundred kilometres for all modes, but larger for modes 1-3 and smaller for 4-7. These are small enough values to allow for significant wave growth while the wave stays in the electron energisation region, whose length can be estimated to be at least $1 R_{E}(6372 \mathrm{~km})$. The perpendicular $e$-folding distance is $10-20 \mathrm{~km}$ for modes $1-3$, and below $3 \mathrm{~km}$ for modes $4-7$. These values must be divided by 7 when projecting them down to the ionosphere. If one requires seven e-foldings for significant growth and assumes that a wave propagates perpendicular to an auroral arc, the perpendicular $e$-folding distances given in the table thus correspond directly to the smallest possible arc width in the ionosphere that can accommodate the waves.

The parallel phase velocities for the growing modes in Table 1 are higher than thermal electron velocities, which probably explains why these modes are growing and thus not too heavily Landau-damped by the electrons. Higher Bernstein modes $(>7)$ are enough Landau-damped so that in the linear theory they have negative growth rates. To have efficient electron energisation due to a Landau resonance, one needs a nonlinear coupling between modes which pumps energy from the linearly unstable modes to some of the linearly 


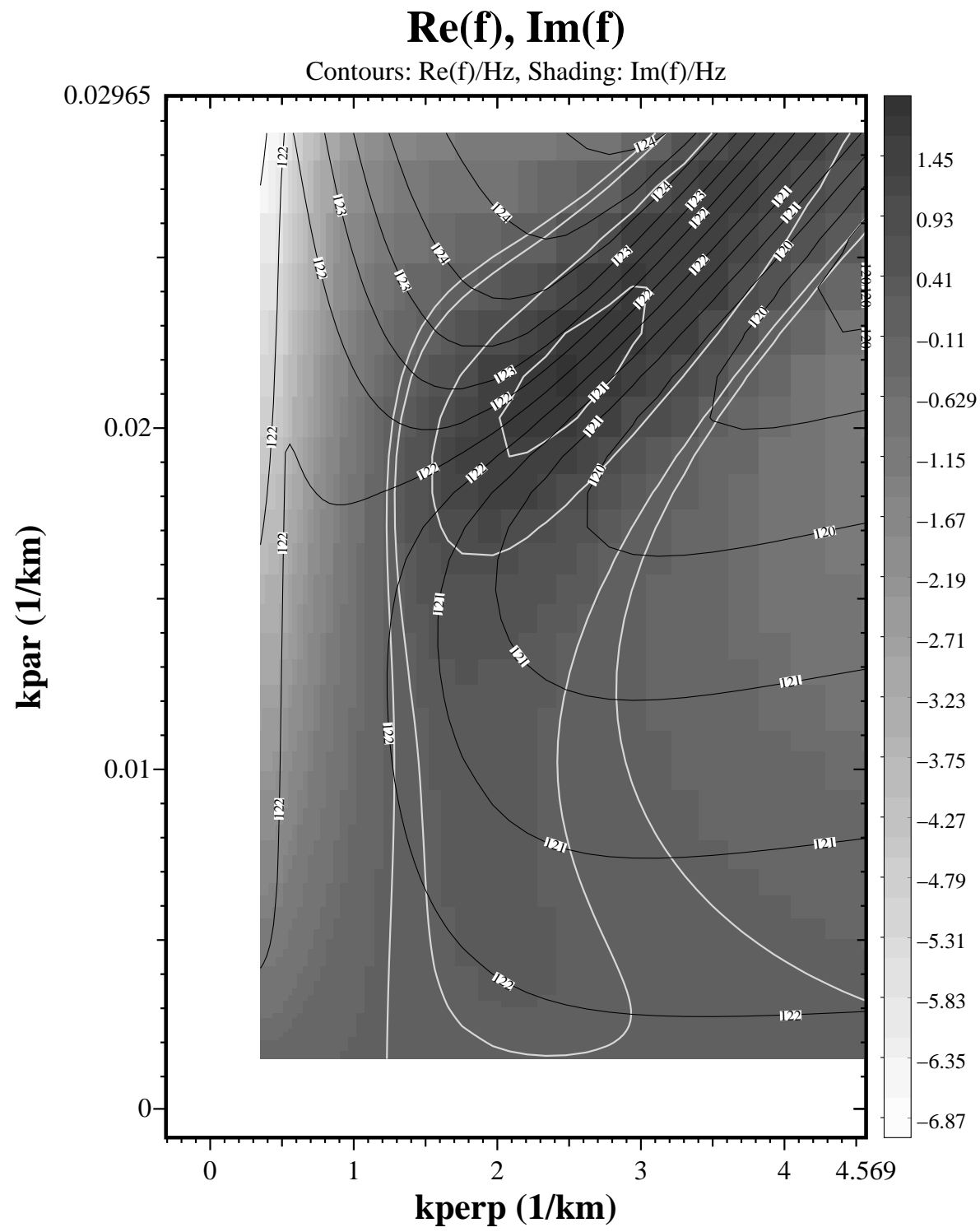

Fig. 6. Frequency (black labelled contours) and temporal growth rate (shading) for seventh Bernstein mode. White contours are drawn at $0 \%, 10 \%, 50 \%$, and $90 \%$ of the maximum growth rate. The wave vector $k=2 \pi / \lambda$ is in units of $\mathrm{km}^{-1}$. Here, $k=0.1 \mathrm{~km}^{-1}$ corresponds to $k \rho_{i}=1$, where $\rho_{i}=10 \mathrm{~km}$ is the Larmor radius of the shell distribution $(5 \mathrm{keV})$ protons. damped ones, which then accelerate the electrons. This is the subject of the next section where a numerical simulation is considered.

\section{Numerical simulation of Bernstein waves}

To study the nonlinear effects and mode coupling we use a two-dimensional (2-D) electrostatic particle-in-cell (PIC) simulation. We use an explicit particle code (Birdsall and Langdon, 1985; Hockney and Eastwood, 1988) where ions are treated using the Buneman solver (Hockney and Eastwood, 1988) and the guiding centre approximation is used for the electrons. The electrons thus behave one-dimensionally and respond only to the parallel component of the electric field. This approximation is not essential but speeds up the simulation; it is valid in the region we are considering $(\sim 18000 \mathrm{~km}$ altitude, $B=1000 \mathrm{nT})$, since the electron
Larmor radius is the same or smaller than the electron Debye length. A rather similar simulation technique but without using the guiding centre approximation for the electrons has been used in the acceleration region to study ion waves generated by ion beams (Mottez and Chanteur, 1991; Mottez et al., 1992).

In the 2-D simulation, $x$ is the perpendicular and $z$ is the field-aligned coordinate. Electron $\boldsymbol{E} \times \boldsymbol{B}$ drift occurs in the homogeneous $y$ direction and, therefore, can be ignored. The number of grid cells is 2048 in the parallel and 128 in the perpendicular direction. The number of ions per grid cell is 100. The timestep is $0.0142 \mathrm{~ms}$, corresponding to $0.8 \omega_{p e}^{-1}$. The simulation is parallelised using the Message Passing Interface (MPI) version 1.2. The duration of the simulation is $0.25 \mathrm{~s}$. Linear weighting is used, and to reduce numerical heating further, spatial smoothing is employed by effectively averaging over 2 cells in the perpendicular and 8 cells in the parallel direction when computing the charge density used 


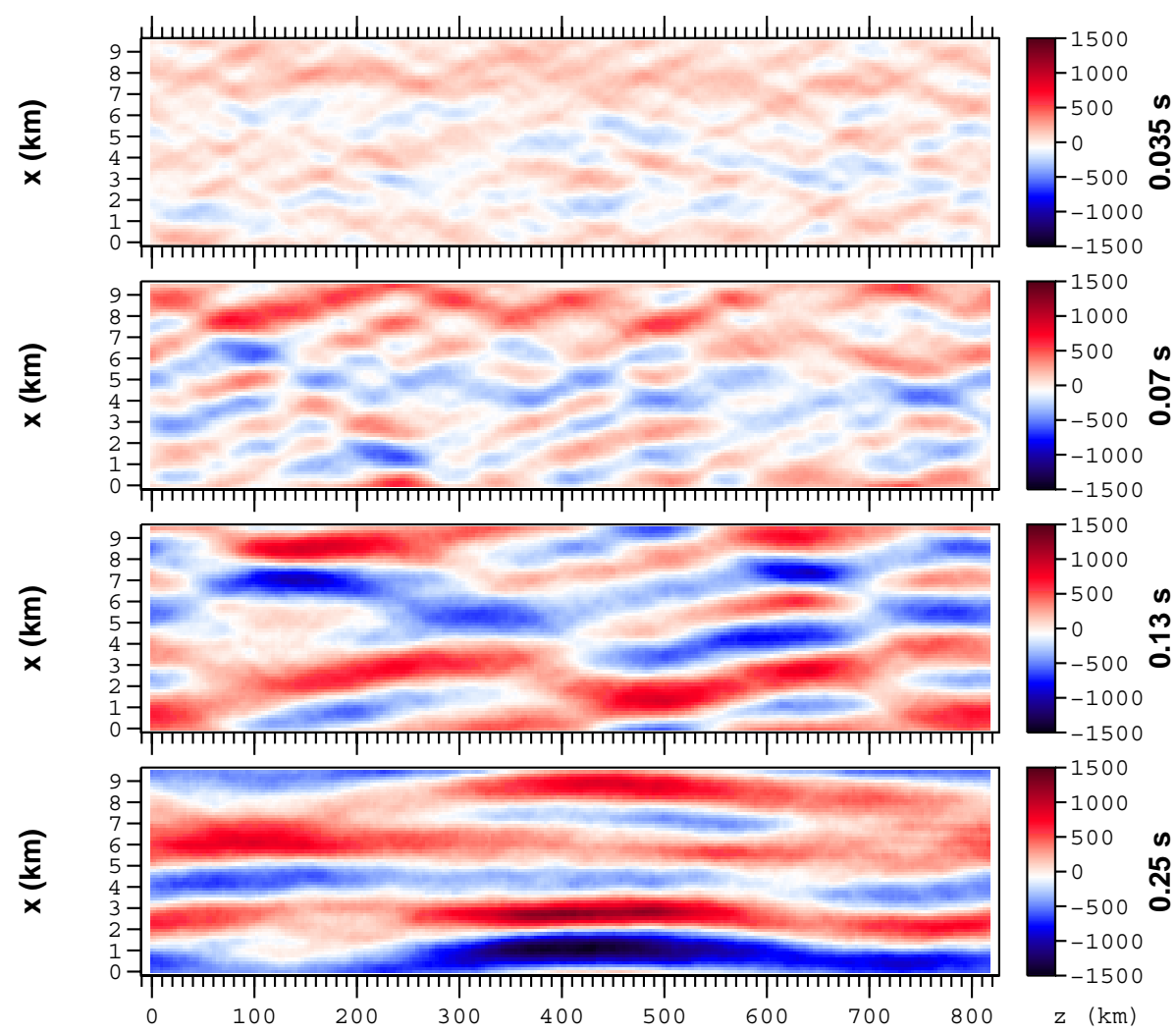

Fig. 7. Electric potential in the shell distribution simulation at $0.035 \mathrm{~s}$ (top), $0.07 \mathrm{~s}$ (second panel), $0.13 \mathrm{~s}$ (third panel) and $0.25 \mathrm{~s}$ (bottom panel). The potential has been averaged over 36 time steps $(0.5 \mathrm{~ms})$ in each case.

for Poisson's equation.

To model a shell distribution in the simulation in a simple way, we take an isotropic proton Maxwellian with $5 \mathrm{keV}$ thermal energy and $0.7 \mathrm{~cm}^{-3}$ density and cut out the part which is below the thermal energy. This produces a shell whose details differ from the one used in WHAMP computations (which was a difference of two Maxwellians), but we shall show that the results are nevertheless rather similar except that the growth rates in the simulation are higher. A cold ion population with density $0.3 \mathrm{~cm}^{-3}$ and temperature 1 $\mathrm{eV}$ is used as in WHAMP. The initial electron temperature is taken to be $100 \mathrm{eV}$ to facilitate the study of electron energisation (if a lower temperature was chosen, numerical heating would become more of a problem). To evaluate the effect of numerical heating, we perform, in addition to the shell distribution run, a baseline run which is otherwise identical to the shell run, but the hot proton shell distribution is replaced by an isotropic Maxwellian $5 \mathrm{keV}$ population.

The electric potential in the shell distribution run is shown in Fig. 7 at four times. The first time $(0.035 \mathrm{~s})$ corresponds to the state where the Bernstein waves that are linearly the most unstable have appeared and are growing linearly. At $0.07 \mathrm{~s}$ (second panel) they have intensified but still behave linearly and the spectrum is dominated by rather short wavelengths (a few kilometres in perpendicular). At $0.13 \mathrm{~s}$ (third panel) nonlinear phenomena have started to take over and wave power is moving towards longer wavelengths in $k$-space (inverse cas- cade process). The cascade process continues and at $0.25 \mathrm{~s}$ (bottom panel) the situation at the end of the run is seen.

\subsection{Electric field spectrum}

Figures 8 and 9 shows the spatiotemporal $\left(k_{x}, \omega\right)$ power spectrum of the perpendicular electric field fluctuations in the baseline and shell runs, respectively. Different Bernstein modes appear as a series of peaks forming an oblique line near the left side of Fig. 9. Waves near the lower hybrid frequency form a more horizontally aligned feature slightly above $100 \mathrm{~Hz}$. These waves appear at higher $k_{\perp}$ than the Bernstein waves. There is another, weaker branch of waves at about $50 \mathrm{~Hz}$ whose origin is not clear to us, appearing in both the baseline and shell runs.

The time series of the perpendicular electric field $E_{x}$ in both runs is shown in Fig. 10. The thermal noise in the baseline run has an amplitude of about $\pm 200 \mathrm{mV} / \mathrm{m}$, while the Bernstein waves in the shell distribution run reach amplitudes of about $\pm 700 \mathrm{mV} / \mathrm{m}$. Electric fields of several hundred $\mathrm{mV} / \mathrm{m}$ are observed in some events, though not in the one that was shown in Fig. 2; this is not a problem for interpretation since the simulation uses an idealised shell distribution and thus produces strong emissions. From the temporal power spectrum of $E_{x}$ (Fig. 11) we see that the Bernstein modes are clearly distinguishable from the thermal noise. Not every Bernstein mode is excited, which may be due to the periodic 


\section{Ex spectrum, Baseline run}

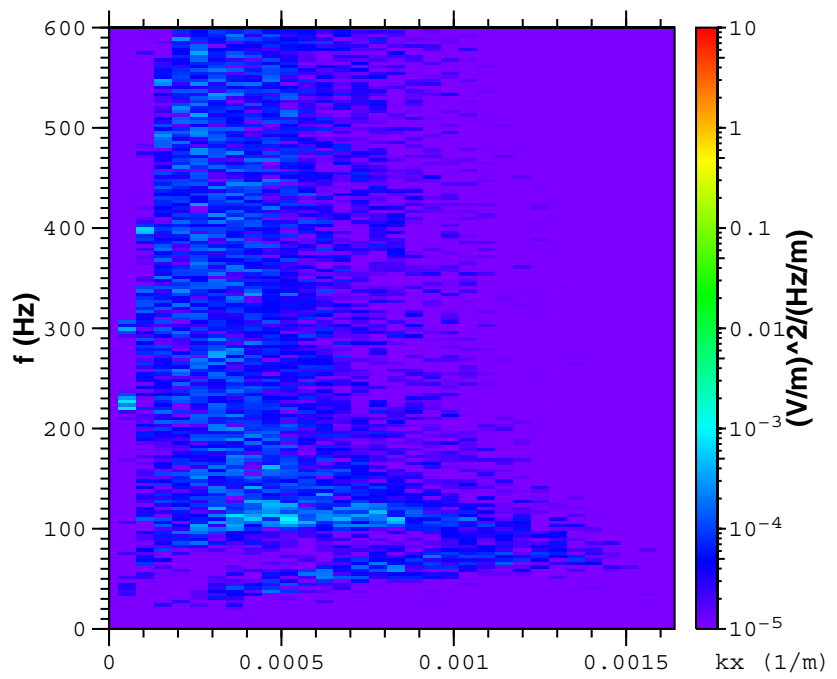

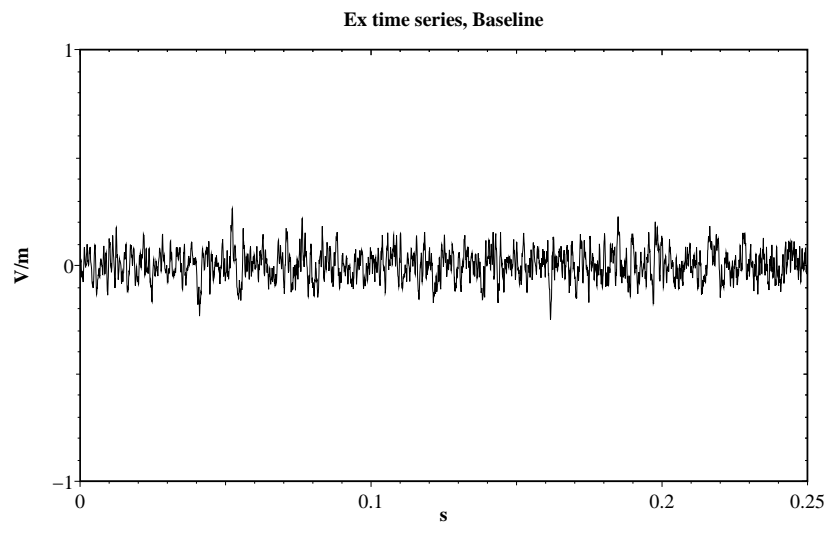

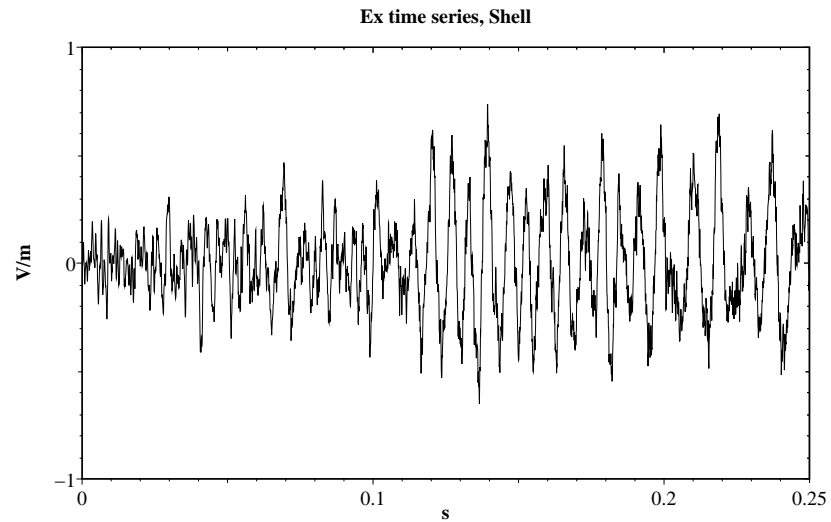

Fig. 10. Time series of the perpendicular electric field $E_{x}$ at one $z$ value in baseline run (upper panel) and shell distribution run (lower panel).

quency where the power maximises moves up if we make the box smaller.

\subsection{Parallel electron energisation by Bernstein waves}

Figures 12 and 13 show the history of the parallel and perpendicular temperature, as well as electrostatic energy per electron of the plasma populations in the baseline and shell distribution runs, respectively.

In the baseline run, electrons are heated from $100 \mathrm{eV}$ to $145 \mathrm{eV}$ in $0.25 \mathrm{~s}$. This energisation is due to thermal fluctuations and numerical heating (Birdsall and Langdon, 1985). In the shell distribution run, the electron heating is from 100 to $181 \mathrm{eV}$.

The perpendicular cold ion energy is increased in the baseline run from $1 \mathrm{eV}$ to $2.1 \mathrm{eV}$ and from $1 \mathrm{eV}$ to $10 \mathrm{eV}$ in the shell distribution run. The hot ion perpendicular energy decreases by $30 \mathrm{eV}$ in the baseline run. The parallel energy decreases by $1.5 \mathrm{eV}$. In the shell distribution run the perpendicular energy decreases by $60 \mathrm{eV}$ and the parallel by $2 \mathrm{eV}$. The parallel cold ion energy stays very nearly constant in both runs. The electrostatic wave energy per electron is negligible $(0.2 \mathrm{eV})$ in the baseline run and about $3.7 \mathrm{eV}$ at the end of the shell distribution run. 


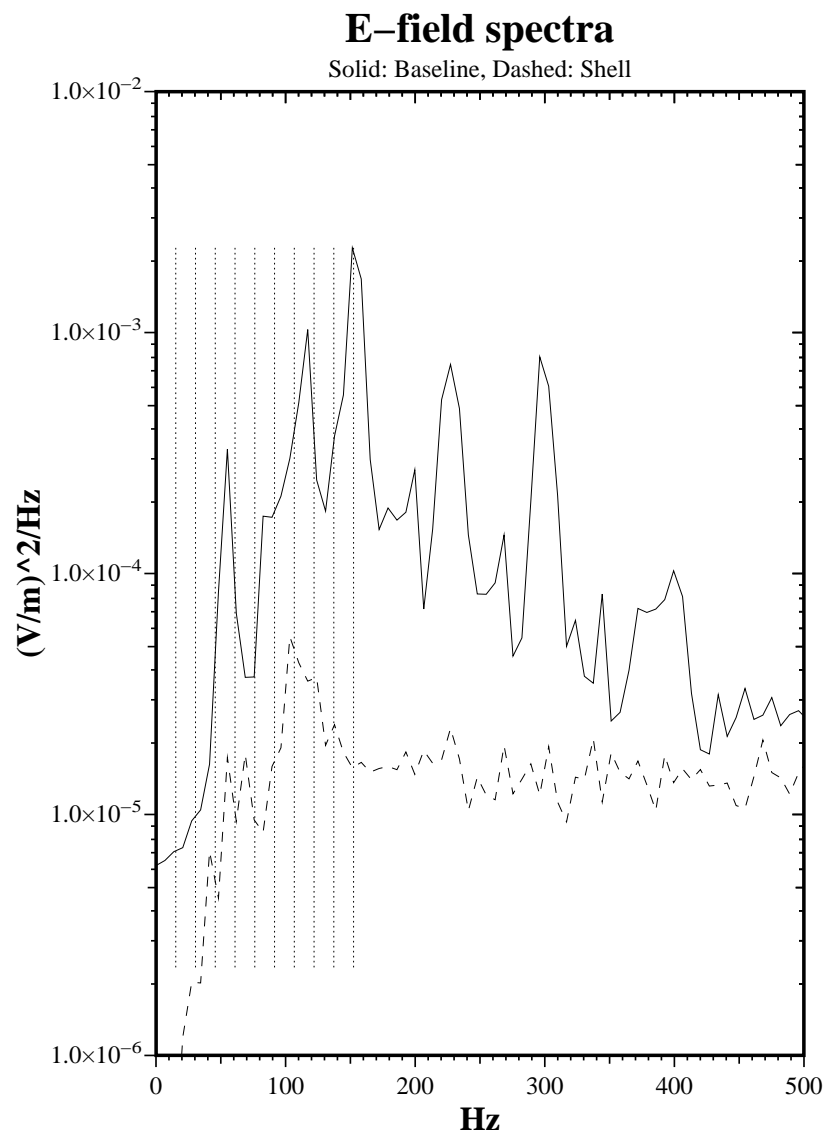

Fig. 11. Temporal power spectrum of perpendicular electric field $E_{x}$ fluctuations averaged over all $z$ in baseline (dashed) and shell distribution runs (solid). Some proton cyclotron harmonic multiples are denoted by dotted vertical lines.

To check the energy budget, recall that the cold ion density is $0.3 \mathrm{~cm}^{-3}$ and the hot ion density is $0.7 \mathrm{~cm}^{-3}$. The energy density of a population with density $n$ is $n T_{\|} / 2+n T_{\perp}$. Calculating the energy budget we find that in the baseline run there is $21.5 \mathrm{eV}$ of energy coming from the hot ions and 1.3 $\mathrm{eV}$ from numerical heating. This energy goes mainly (22.5 $\mathrm{eV}$ ) to electrons, while hot ions receive only $0.3 \mathrm{eV}$. In the shell distribution run, $42.7 \mathrm{eV}$ comes from hot ions and 4.2 $\mathrm{eV}$ from numerical heating. Again, most of the energy (40.5 $\mathrm{eV}$ ) goes to electrons; cold ions take $2.7 \mathrm{eV}$ and the waves $3.7 \mathrm{eV}$. These numbers are computed per electron and per the duration of the run $(0.25 \mathrm{~s})$. By "numerical heating" we refer to the imbalance of the total energy budget. Besides pure numerical heating, there is also a transfer of energy from the hot ions to electrons and cold ions which is caused by the thermal fluctuations of the wave field. This transfer of energy can also be considered numerical in origin in the sense that in reality, the thermal fluctuations are much smaller than in the model.

By subtracting the heating rates of the baseline run from those of the shell run and making the reasonable assumption that the wave energy eventually goes into heating the parti-

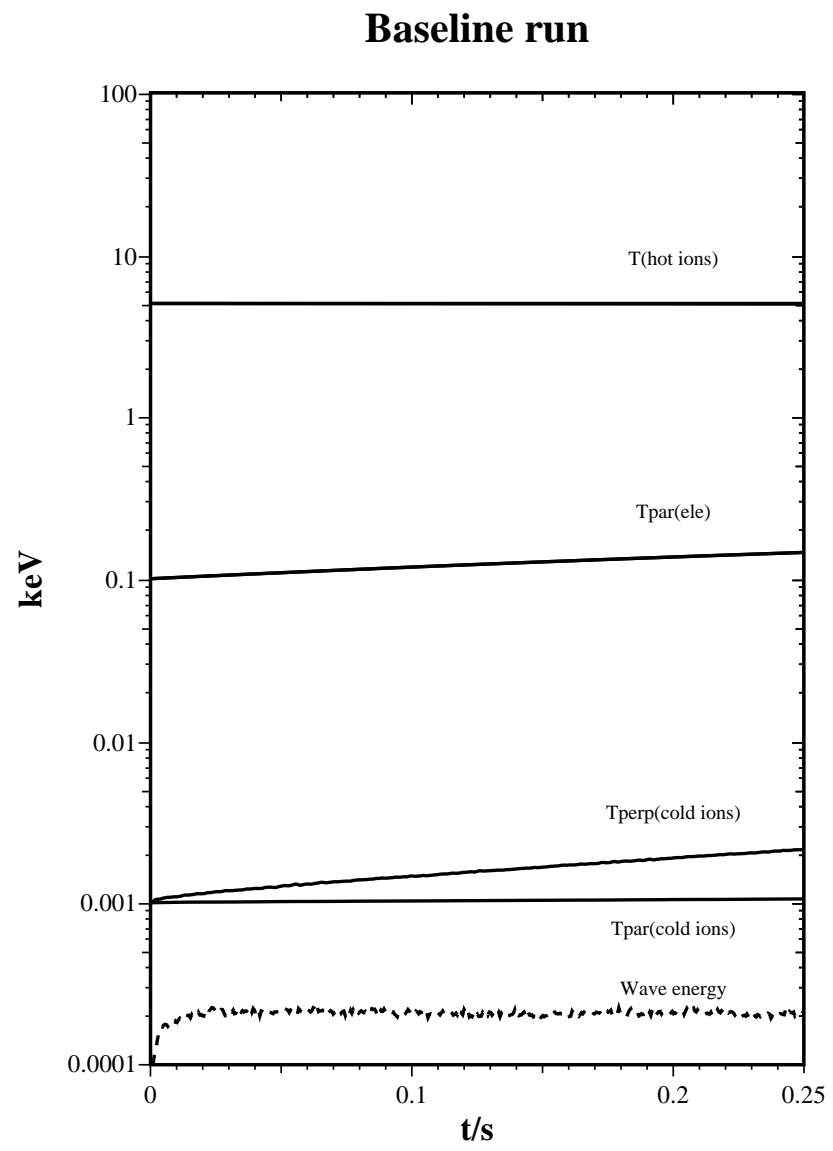

Fig. 12. Energy history of the baseline run. The parallel and perpendicular temperature of various plasma populations are marked in the figure. The electrostatic energy per electron is shown by dashed line.

cles, we can summarise the result by saying that about $21 \mathrm{eV}$ (per electron and per $0.25 \mathrm{~s}$ ) is extracted from the shell distribution ions by the Bernstein waves, of which $13 \%$ goes into heating cold ions perpendicularly and $87 \%$ into increasing the parallel energy of electrons.

\section{Discussion and summary}

We now summarise our new findings from our linear and nonlinear calculations concerning shell distributions as free energy sources of Bernstein modes and the associated electron energisation:

1. A hot ion shell distribution contains rather large amounts of free energy and thus, it is a possible candidate for the energy source for waves that would be able to power auroral electron energisation (Fig. 1).

2. Polar/TIMAS ion data show that ion shell distributions sometimes occur on auroral field lines. In our example event the data are also consistent with the hypothesis that the shell distribution provides the free energy for wave-induced parallel electron energisation (Fig. 2). 


\section{Shell distribution run}

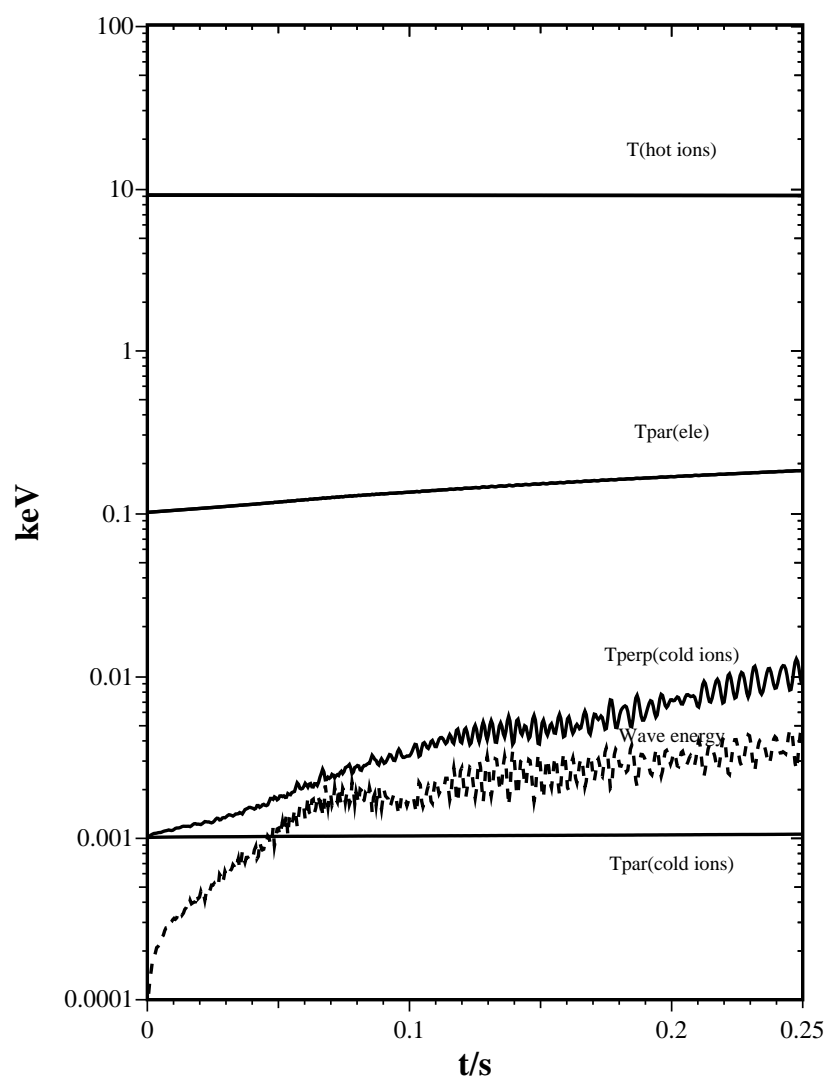

Fig. 13. Energy history of the shell distribution run. Same format as in Fig. 12.

3. For the parameters studied with WHAMP, Bernstein modes 4-7 have large temporal growth rates, and $e$ folding times of down to $0.1 \mathrm{~s}$ can be reached (Table 1).

4. The perpendicular $e$-folding distance is of the order of the auroral arc width (a few kilometres) or smaller when projected to the ionosphere. The parallel $e$-folding distance is not too large (Table 1).

5. According to the simulation, the electrons are heated by electrostatic waves by $\sim 20 \mathrm{eV}$ in $0.25 \mathrm{~s}$. The cold ions receive about $10 \%$ of the heating. Electrons receive parallel and ions receive perpendicular energisation (Fig. 13).

6. The simulation shows an inverse cascade process for the Bernstein waves to operate, i.e. a transfer of energy from short to long wavelengths by nonlinear mechanisms (Fig. 7).

We now discuss how the results could be applied in a model for auroral electron acceleration and inverted- $\mathrm{V}$ region formation.

It has been suggested (Janhunen and Olsson, 2000) that an O-shaped negative potential structure can accelerate electrons in cooperation with waves and that the existence of a potential structure can actually be a consequence of waveinduced parallel energisation of electrons (the "cooperative model"). For this model to work, sufficient wave power energising electrons in the parallel direction are needed in the topside of the potential structure. It should be stressed that in this model, the waves are not responsible for generating the shape of the low-altitude inverted- $\mathrm{V}$ peaked electron distribution, but only for increasing the parallel energy of the electrons in the upper part of the potential structure. It is also not required that the waves carry a large (or any) downward Poynting flux if they are generated locally. From the simulation we saw that the ion Bernstein waves and their associated electron energisation are at least one promising candidate for the required wave-particle interaction that might complete the scenario for auroral electron acceleration. A confirmation that the broad-band signals seen here are really Bernstein waves is still to be done and requires simulations in a large box with a nonuniform magnetic field and preferably also electromagnetic terms included. Observationally the task is very difficult if it is so that the Doppler shift and nonuniform magnetic field effects turn the Bernstein wave "comb" into a quasi-continuous, broad-band spectrum, because then there is no specific observational feature that could be linked with Bernstein waves.

Acknowledgements. We thank Mats André and Bengt Eliasson for useful discussions. We are grateful to F.S. Mozer for EFI data and C.A. Kletzing for HYDRA data. The computations were carried out in a parallel computer at the Department of Scientific Computing of Uppsala University. The work of PJ was supported by the Academy of Finland and that of AO by the Swedish Research Council.

Topical Editor G. Chanteur thanks two referees for their help in evaluating this paper.

\section{References}

André, M.: Dispersion surfaces, J. Plasma Phys., 33, 1-19, 1985.

André, M.: Electrostatic ion waves generated by ion loss-cone distributions in the magnetosphere, Ann. Geophysicae, 4, 241-246, 1986.

André, M., Temerin, M., and Gorney, D.: Resonant generation of ion waves on auroral field lines by positive slopes in ion velocity space, J. Geophys. Res., 91, 3145-3151, 1986.

Ashour-Abdalla, M. and Thorne, R. M.: Toward a unified view of diffuse auroral precipitation, J. Geophys. Res., 83, 4755-4766, 1978.

Barbosa, D. D.: Stability of shell distributions, Planet. Space Sci., 25, 981-984, 1977.

Bingham, R., Bryant, D. A., and Hall, D. S.: Auroral electron acceleration by lower-hybrid waves, Ann. Geophysicae, 6, 159-168, 1988.

Bingham, R., Kellett, B. J., Cairns, R. A., Dendy, R. O., and Shukla, P. K.: Wave generation by ion horseshoe distributions on auroral field lines, Geophys. Res. Lett., 26, 2713-2716, 1999.

Birdsall, C. K. and Langdon, A. B.: Plasma physics via computer simulation, McGraw-Hill Book Company, Singapore, 1985.

Bryant, D. A., Cook, A. C., Wang, Z.-S., De Angelis, U., and Perry, C. H.: Turbulent acceleration of auroral electrons, J. Geophys. Res., 96, 13 829-13 839, 1991. 
Bryant, D. A. and Perry, C. H.: Velocity-space distributions of wave-accelerated auroral electrons, J. Geophys. Res., 100, 23711-23 725, 1995.

Bryant, D. A.: Electron acceleration in the aurora and beyond, Institute of Physics Publishing, Bristol 1999.

Carlqvist, P. and Boström, R.: Space-charge regions above the aurora, J. Geophys. Res., 75, 7140-7146, 1970.

Evans, D. S.: Precipitating electron fluxes formed by a magnetic field aligned-potential difference, J. Geophys. Res., 79, 28532858, 1974.

Elphic, R. C. and Gary, S. P.: ISEE observations of low frequency waves and ion distribution function evolution in the plasma sheet boundary layer, Geophys. Res. Lett., 17, 2023-2026, 1990.

Freund, H. P. and Wu, C. S.: Stability of spherical shell distribution of pickup ions, J. Geophys. Res., 93, 14 277-14 283, 1988.

Genot, V., Louarn, P., and Mottez, F.: Electron acceleration by Alfvén waves in density cavities, J. Geophys. Res., 105, 27 611$27620,2000$.

Goertz, C. K. and Boswell, R. W.: Magnetosphere-ionosphere coupling, J. Geophys. Res., 84, 7239-7246, 1979.

Gurnett, D. A. and Frank, L. A.: A region of intense plasma wave turbulence on auroral field lines, J. Geophys. Res., 82, 10311050, 1977.

Gurnett, D. A.: Auroral plasma waves, pp. 241-254, in: Auroral physics, (Eds) Meng, C.-I., Rycroft, M. J., and Frank, L. A., Cambridge, 1991.

Harvey, P., Mozer, F. S., Pankow, D., Wygant, J., Maynard, N. C., Singer, H., Sullivan, W., Anderson, P. B., Pfaff, R., Aggson, T., Pedersen, A., Fälthammar, C. G., and Tanskanen, P.: The electric field instrument on the Polar satellite, Space Sci. Rev., 71, 583596,1995

Hockney, R. W. and Eastwood, J. W.: Computer simulation using particles, Adam Hilger, Philadelphia, 1988.

Huddleston, M. M., Pollock, C. J., Wüest, M. P., Pickett, J. S., Moore, T. E., and Peterson, W. K.: Toroidal ion distributions observed at high altitudes equatorward of the cusp, Geophys. Res. Lett., 27, 469-472, 2000.

Janhunen, P. and Olsson, A.: New model for auroral acceleration: O-shaped potential structure cooperating with waves, Ann. Geophysicae, 18, 596-607, 2000.

Kintner, P. M.: On the distinction between electrostatic ion cyclotron waves and ion cyclotron harmonic waves, Geophys. Res. Lett., 7, 585-588, 1980.

Kletzing, C. A.: Electron acceleration by kinetic Alfvén waves, J. Geophys. Res., 99, 11 095-11 103, 1994.

Louarn, P., Wahlund, J.-E., Chust, T., de Feraudy, H., Roux, A.,
Holback, B., Dovner, P. O., Eriksson, A. I., and Holmgren, G.: Observation of kinetic Alfvén waves by the FREJA spacecraft, Geophys. Res. Lett., 21, 1847-1850, 1994.

Mottez, F. and Chanteur, G.: Nonlinear bidimensional evolution of ion beam driven electrostatic instabilities in the auroral region, J. Geophys. Res., 96, 21 141-21 147, 1991.

Mottez, F., Chanteur, G., and Roux, A.: Filamentation of plasma in the auroral region by an ion-ion instability: a process for the formation of bidimensional potential structures, J. Geophys. Res., 97, 10 801-10 810, 1992.

Roth, I. and Hudson, M. K.: Lower hybrid heating of ionospheric ions due to ion ring distributions in the cusp, J. Geophys. Res., 90, 4191-4203, 1985.

Rönnmark, K.: WHAMP - Waves in Homogeneous Anisotropic Multicomponent Plasmas, KGI Report 179, Kiruna Geophysical Institute, Kiruna, 1982.

Scudder, J. D., Hunsacker, F., Miller, G., Lobell, J., Zawistowski, T., Ogilvie, K., Keller, J., Chornay, D., Herrero, F., Fitzenreiter, R., et al.: Hydra: A 3-dimensional electron and ion hot plasma instrument for the Polar spacecraft of the GGS mission, Space Sci. Rev., 71, 459-495, 1995.

Sentman, D. D., Leboeuf, J. N., Katsouleas, T., Huff, R. W., and Dawson, J. M.: Electrostatic instabilities of velocity-space-shell distributions in magnetized plasmas, Phys. Fluids, 29, 25692579, 1986.

Shelley, E. G., Ghielmetti, A. G., Balsiger, H., Black, R. K., Bowles, J. A., Bowman, R. P., Bratschi, O., Burch, J. L., Carlson, C. W., Coker, A. J., Drake, J. F., Fischer, J., Geiss, J., Johnstone, A., Kloza, D. L., Lennartsson, O. W., Magoncelli, A. L., Paschmann, G., Peterson, W. K., Rosenbauer, H., Sanders, T. C., Steinacher, M., Walton, D. M., Whalen, B. A., and Young, D. T.: The Toroidal Imaging Mass-Angle Spectrograph (TIMAS) for the Polar mission, Space Sci. Rev., 71, 497-530, 1995.

Stasiewicz, K., Bellan, P., Chaston, C., Kletzing, C., Lysak, R., Maggs, J., Pokhotelov, O., Seyler, C., Shukla, P., Stenflo, L., Streltsov, A., and Wahlund, J.-E.: Small Scale Alfvénic Structure in the Aurora, Space Sci. Rev., 92, 423-533, 2000.

Volwerk, M., Louarn, P., Chust, T., Roux, A., and de Feraudy, H.: Solitary kinetic Alfvén waves: a study of the Poynting flux, J. Geophys. Res., 101, 13 335-13 343, 1996.

Wygant, J. R., Keiling, A., Cattell, C. A., Johnson, M., Lysak, R. L., Temerin, M., Mozer, F. S., Kletzing, C. A., Scudder, J. D., Peterson, W., et. al.: Polar spacecraft based comparisons of intense electric fields and Poynting flux near and within the plasma sheet-tail lobe boundary to UVI images: an energy source for the aurora, J. Geophys. Res., 105, 18 675-18 692, 2000. 